\title{
AVD Regimen
}

National Cancer Institute

\section{Source}

National Cancer Institute. AVD Regimen. NCI Thesaurus. Code C140093.

A reg imen consisting of doxorubicin, vinblastine and dacarbazine that can be used for the treatment of Hodgkin lymphoma (HL). 